\title{
Emerging Therapies in Ischaemic Stroke Management
}

\author{
a report by \\ Turgut Tatlisumak and Markku Kaste
}

Department of Neurology, Helsinki University Central Hospital

\section{Pharmaceutical Recanalisation Therapies}

When given within three hours of stroke onset in well-selected eligible patients, intravenous (IV) thrombolysis with realtime plasminogen activator (rt-PA) has become the cornerstone of hyperacute stroke therapy. This follows approvals by the US Food and Drug Administration (FDA) in 1996 and the European Medicines Agency (EMEA) in 2002. Although effective, the therapy has several limitations, including certain contraindications, a short application time window, fear of haemorrhagic complications, which may lead to physicians refraining from using rt-PA, the lack of the necessary health service infrastructure in many developed countries and the fact that not all patients benefit from IV thrombolysis. Recanalisation rates with IV thrombolysis range between 20 and $66 \%$ (average $46 \%$ for all occlusions) depending on the occlusion site, size and consistency of the thromboembolic material and several other factors. Even if recanalisation is achieved, reocclusion associated with clinical deterioration may occur

Only $4-5 \%$ of all ischaemic stroke patients in the developed world receive IV thrombolysis. According to the Safe Implementation of Thrombolysis in Stroke-Monitoring Study (SITS-MOST) registry, in Finland more ischaemic stroke patients receive thrombolysis per million inhabitants than in any other EU country, and the emergency room of Helsinki University Central Hospital provides more thrombolysis than any other European hospital. However, even in Finland stroke patients do not have equal opportunities for thrombolysis. For benefits to be achieved, a number needed to treat (NNTT) of seven ${ }^{2}$ was determined, and for hazards to occur, a number needed to harm (NNTH) of one in $100^{3}$ has been calculated.

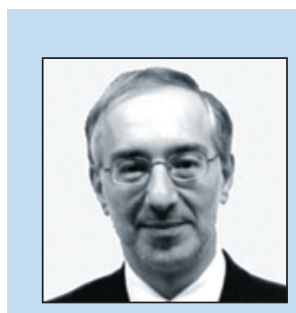

Turgut Tatlisumak is an Associate Professor and Vice Chairman of the Department of Neurology at Helsinki University Central Hospital. He leads the Acute Stroke Unit and his research is focused on clinical and experimental stroke. Dr Tatlisumak has participated in over 20 international multicentre acute stroke trials, including thrombolytic therapy trials. He is currently establishing a national telestroke consultation network in Finland.

E: turgut.tatlisumak@hus.fi

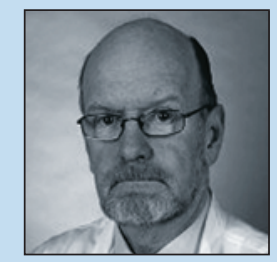

Markku Kaste is Chief Neurologist in the Department of Neurology at Helsinki University Central Hospital and Chairman of the Department of Neuroscience at the University of Helsinki. He is Vice President of the World Stroke Organization (WSO) and Chairman of the Finnish Neurological Society (FNS). He was an Associate Editor of the European edition of Stroke. Professor Kaste has been recognised by numerous prestigious awards, including the Johan Jacob Wepfer Award 2006 and the Karolinska Stroke Award 2006.
The earlier thrombolysis is given, the better the outcome; however, not all stroke patients arrive early enough and thus the current three-hour time window is often too short. In addition to making thrombolysis available for more patients, several other treatments need to be developed. A recent analysis suggested that the benefit of IV thrombolysis continues up to 4.5 hours. ${ }^{4}$ The European Co-operative Acute Stroke Study III (ECASS III) is a multinational, controlled, randomised study in which patients are randomised to placebo or standard IV rt-PA in a time-frame of three to 4.5 hours from stroke onset. ECASSS III will bring more certainty to the question of whether patients benefit from IV thrombolysis between three and 4.5 hours after stroke. Attempts have been made to further extend the time window for IV thrombolysis with the use of modern brain imaging modalities such as diffusion-perfusion magnetic resonance imaging (MRI) and perfusion computed tomography, with promising success. These modalities may help patient selection for thrombolysis after three hours after the onset of symptoms.

Intra-arterial (IA) thrombolysis may be more effective than IV thrombolysis, especially in cases of large artery occlusions such as internal carotid, basilar or proximal middle cerebral artery occlusions with extensive thrombus. IA thrombolysis leads more often to recanalisation compared with IV thrombolysis (63 versus $46 \%$ for all vessels) and is associated with a similar rate of intracranial haemorrhages to IV thrombolysis. ${ }^{1}$ IA thrombolysis with pro-urokinase was beneficial even up to six hours after stroke onset in one study, ${ }^{5}$ but it was not approved by the FDA. A combined approach of IV and IA thrombolysis in open trials has been found to be better than IV thrombolysis, with a similar haemorrhagic complication rate to IV thrombolysis alone.6,7 A large randomised trial is warranted.

Novel thrombolytic agents including desmoteplase, tenecteplase and reteplase have longer half-lives, higher fibrin specificity and a higher resistance to PA inhibitor-1. Originally isolated from vampire bat saliva, IV desmoteplase was given in randomised trials three to nine hours after stroke onset to patients with a substantial region of penumbra. The first two trials delivered promising results, 8,9 but the most recent efficacy trial could not confirm their results. A mutant form of rt-PA, tenecteplase, was found to be safe in a small study when given IV at $0.1-0.5 \mathrm{mg} / \mathrm{kg}$ within three hours of stroke onset. ${ }^{10} \mathrm{~A}$ randomised phase $\mathrm{llb}$ trial comparing three different doses of tenecteplase with standard IV rt-PA within three hours of the time window is under way. Reteplase can be administered as a bolus, and was superior to standard rt-PA in myocardial infarction. Experience with reteplase in stroke patients remains limited.

\section{Mechanical Recanalisation}

Mechanical thrombus retraction has been used as a rescue therapy for patients who do not respond to thrombolytics, or as a primary treatment for those with contraindications to thrombolytics, and may lead to higher 
rates of vessel patency. Thromboemboli that are especially platelet-poor may be more amenable to mechanical thrombectomy and remain intact during the procedure. Mechanical thrombectomy can be combined with IA thrombolytic agents when appropriate, although it may increase the intracranial haemorrhage rate. A catheter can be used to aspirate clots, in a procedure in which a capable interventional radiologist is needed. This process requires large-bore catheters to allow adequate suction and therefore is limited to most proximal arterial occlusions. Among many emerging devices, the Mechanical Embolus Removal in Cerebral Ischemia device (MERCl, Concentric Medical, Mountain View, California) has received the most attention. A microcatheter is inserted through the embolus to the distal side of the occlusion in a similar way to a corkscrew, and stabilises the thrombus in a nitinol-made wire basket. During thrombus extraction, the balloon in the distal tip of the main catheter is inflated to cease forward blood flow, thus preventing thrombus fragments lodging into distal arteries. The thrombus is then aspirated into the catheter and subsequently removed with the catheter from the arterial tree. A series of clinical trials ${ }^{11-13}$ demonstrated high recanalisation rates in a time window of three to eight hours post-stroke with reasonable haemorrhagic complication rates, and led to FDA approval for endovascular recanalisation of cerebral arteries in patients for whom IV rt-PA is contraindicated or has failed. Angioplasty (with or without stenting) has been used for acute stroke in anecdotal cases with success, but experience is limited.

Ultrasonic energy has been internally delivered to the clot through a catheter (the EKOS MicroLysUS infusion catheter, EKOS Corp, Seattle), causing microscopic cavitations within the thrombus and augmenting the effect of following thrombolytic drug by facilitating its penetration deep in the clot; ${ }^{14}$ studies in this area are ongoing. Similarly, laser energy has been applied locally and internally to the clot material with the Endovascular PhotoAcoustic Recanalization (EPAR) device (Endovasix, Belmont, California). A small study showed not only high recanalisation rates, but also high mortality. ${ }^{15}$ Ultrasonic energy was combined with IV rt-PA in the Combined Lysis of Thrombus in Brain Ischaemia Using Transcranial Ultrasound and Systemic Tissue Plasminogen Activator (CLOTBUST) trial, ${ }^{16}$ which demonstrated significantly higher recanalisation rates in patients who received the combination of IV thrombolysis and transcranial ultrasound for middle cerebral artery occlusions. However, the clinical outcome was not significantly better in the combination-treated group. A variety of microbubbles has been developed to improve recanalisation rates. They can be applied intravenously, and incorporate themselves to the thromboembolus and explode within the clot when predisposed to external ultrasound energy, leading to fragmentation of the clot material. These therapies are under intense investigation.

\section{Hypothermia}

Hypothermia has been proved to be neuroprotective in numerous experimental studies and hyperthermia is associated with poor outcome in clinical studies. Two randomised trials 17,18 have shown that reducing body temperature to $32^{\circ} \mathrm{C}$ from $34^{\circ} \mathrm{C}$ for $12-24$ hours in comatose survivors of cardiac arrest leads to better neurological outcomes and reduced mortality. Moderate hypothermia in stroke patients has been tried in a small patient series; ${ }^{19}$ however, a well-controlled randomised trial is required. Deep hypothermia necessitates anaesthesia and respiratory care with intubation and therefore has many severe complications and requires extensive resources, which are not always available. In contrast, mild hypothermia with invasive or non-invasive systems may be accomplished in awake patients and can easily be given in stroke units. Pilot studies have shown a feasibility of mild $\left(34-36^{\circ} \mathrm{C}\right)$ and moderate $\left(32-34^{\circ} \mathrm{C}\right)$ hypothermia in stroke. Randomised studies are ongoing to prove whether mild or moderate hypothermia is beneficial in acute stroke patients.

\section{Craniectomy}

Following a number of experimental and non-randomised pilot clinical studies, three large, randomised, controlled clinical trials - Decompressive Craniectomy In Malignant Middle Cerebral Artery Infarcts (DECIMAL), Decompressive Surgery for the Treatment of Malignant Infarction of the Middle Cerebral Artery (DESTINY) and the Hemicraniectomy After Middle Cerebral Artery Infarction with Life-Threatening Edema Trial (HAMLET) were initiated to test the safety and efficacy of decompressive craniectomy in malignant middle cerebral artery infarction in relatively young ischaemic stroke patients (18-60 years of age) even up to 48 hours after stroke onset. When the results of these three studies were pooled, they revealed a reduced death rate and improved functional recovery without increasing the proportion of severely disabled patients. ${ }^{20}$ Decompressive craniectomy is already in use in comprehensive stroke centres.

\section{Blood-pressure-increasing/-decreasing Treatments}

Induced hypertension may increase cerebral perfusion in penumbral regions and alleviate ischaemic injury. Induced hypertension by means of drugs (phenylephrine) or invasive devices such as the NeuroFlo (CoAxia, Maple Grove, Minnesota) is under evaluation. A reduction of $10 \mathrm{mmHg}$ in arterial systolic blood pressure was associated with an $18 \%$ increase in mortality in a post hoc analysis of the International Stroke Trial, ${ }^{21}$ but in the Acute Candesartan Cilexetil Therapy in Stroke Survivors (ACCESS) Study the treatment to reduce blood pressure had a positive effect on the outcome. ${ }^{22}$ Further trials in which blood pressure is reduced to improve the outcome of patients are under way.

\section{Neuroprotective Treatments}

Neuroprotective therapy is appealing because neuroprotective agents can be safely started without a brain scan even in an ambulance and can be combined with various other therapies, and therefore may help to extend the time window for thrombolysis. A plethora of agents has been tested in randomised clinical trials. So far not one has been proved effective. Recent success with NYX-059 in the first Stroke Acute Ischemic NXY-059 Trial (SAINT) was encouraging, ${ }^{23}$ but the second trial had negative results, ${ }^{24}$ reducing the enthusiasm raised by the first trial. Among many molecules, we can list albumin, statins, citicoline, caffeinol, alpha-amino-3-hydroxy-5methyl-4-isoxazolepropionic acid (AMPA) and n-methyl d-aspartate (NMDA) receptor antagonists, free radical scavengers, anti-inflammatory agents and a number of growth factors and several haematopoietic agents that are already in or about to undergo clinical testing. A comprehensive list of ongoing clinical stroke trials can be seen at www.strokecenter.org/trials/

\section{Brain Plasticity and Stem Cell Therapy}

In experimental stroke, it has been known for a while that a brain damaged by stroke has the capacity to recover using its own or transplanted neural stem cells. This is one of the most exciting frontiers in stroke research, if not the most interesting emerging therapy for stroke. ${ }^{25,26}$ Bone marrow stem cells and drugs such as statins and phosphodiesterase inhibitors can induce neurogenesis from endogenous stem cells and promote functional recovery after experimental stroke. ${ }^{27-29}$ It is hard to predict when stem cell therapies will become part of the recovery-enhancing therapy armoury for stroke patients because much time-intensive basic and clinical research is 
needed. However, two small clinical trials in human stroke patients have been undertaken with FDA approval,30,31 and new ones are imminent. The research field is promising and will probably be included in the next scientific strategy of the European Commission (EC), as the European experts included it in their priority list to the EC. ${ }^{32}$

\section{Combination Therapies}

Combination therapies are becoming more popular. Combined IV and IA thrombolysis, thrombolysis with hypothermia, ultrasound, mechanical clot extraction, antiplatelet agents, neuroprotective agents and other combinations are all feasible and some are already under evaluation.
1. Rha J, Saver J, The impact of recanalization on ischemic stroke outcome: a meta-analysis, Stroke, 2007;38:967-73.

2. The National Institute of Neurological Disorders and Stroke rt-PA Stroke Study Group, Tissue plasminogen activator for acute ischemic stroke, $N$ Engl I Med, 1995;333:1581-7

3. Saver J, Hemorrhage after thrombolytic therapy for stroke: the clinically relevant number needed to harm, Stroke, 2007;38: 2279-83.

4. The Atlantis, ECASS, and NINDS rt-PA Study Group Investigators. Association of outcome with early stroke treatment: pooled analysis of ATLANTIS, ECASS, and NINDS rt-PA stroke trials, Lancet, 2004;363:768-74.

5. Furlan A, Higashida R, Wechsler L, et al., Intra-arterial prourokinase for acute ischemic stroke. The PROACT II Study: a randomized controlled trial. Prolyse in Acute Cerebral Thromboembolism, JAMA, 1999;282:2003-11.

6. IMS Study Investigators, Combined intravenous and intraarterial recanalization for acute ischemic stroke: the Interventional Management of Stroke Study, Stroke, 2004;35: 904-11.

7. The IMS II Trial Investigators, The Interventional Management of Stroke (IMS) II Study, Stroke, 2007;38:2127-35.

8. Furlan A, Eyding D, Albers $G$, et al., Dose Escalation of Desmoteplase for Acute Ischemic Stroke (DEDAS): evidence of safety and efficacy 3 to 9 hours after stroke onset, Stroke, 2006;37:1227-31.

9. Hacke W, Albers G, Al-Rawi Y, et al., The Desmoteplase in Acute Ischemic Stroke Trial (DIAS): a phase II MRI-based 9-hour window acute stroke thrombolysis trial with intravenous desmoteplase, Stroke, 2005;36:66-73

10. Haley $E$, Lyden $P$, Johnston $K$, et al., A pilot dose escalation safety study of tenecteplase in acute ischemic stroke, Stroke 2005;36:607-12.
11. Flint $A$, Duckwiler $G$, Budzik $R$, et al., Mechanical thrombectomy of intracranial carotid occlusion: pooled results of the MERCI and Multi MERCI Part I trials, Stroke, 2007;38: 1274-80.

12. Gobin Y, Starkman S, Duckwiler G, et al., MERCI 1. A phase 1 study of mechanical embolus removal in cerebral ischemia, Stroke, 2004;35:2848-54.

13. Smith W, Sung G, Starkman S, et al., Safety and efficacy of mechanical embolectomy in acute ischemic stroke: results of the MERCI trial, Stroke, 2005;36:1432-8.

14. Mahon B, Nesbit G, Barnwell S, et al., North American clinical experience with the EKOS MicroLysUS infusion catheter for the treatment of embolic stroke, AJNR Am I Neuroradiol, 2003;24: 534-8.

15. Berlis A, Lutsep H, Barnwell S, et al., Mechanical thrombolysis in acute ischemic stroke with endovascular photoacoustic recanalization, Stroke, 2004:35:1112-16.

16. Alexandrov A, Molina C, Grotta J, et al., Ultrasound-enhanced systemic thrombolysis for acute ischemic stroke, N Engl I Med, 2004:351:2170-78

17. Bernard S, Gray T, Buist M, et al., Treatment of comatose survivors of out-of-hospital cardiac arrest with induced hypothermia, N Engl J Med, 2002;346:557-63.

18. Hypothermia After Cardiac Arrest Study Group, Mild therapeutic hypothermia to improve the neurological outcome after cardiac arrest, N Engl J Med, 2002;346:549-56.

19. Schwab S, Schwarz S, Spranger M, et al., Moderate hypothermia in the treatment of patients with severe middle cerebral artery infarction, Stroke, 1998;30:2461-6.

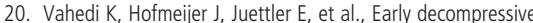
surgery in malignant infarction of the middle cerebral artery: a pooled analysis of three randomised controlled trials, Lancet Neurol, 2007:6:215-22.

21. Leonardi-Bee J, Bath P, Phillips S, et al., Blood pressure and clinical outcomes in the International Stroke Trial, Stroke 2002;33:1315-20.

22. Schrader J, Lüders S, Kulschewski $A$, et al., The ACCESS Study: Evaluation of acute candesartan cilexetil therapy in stroke survivors, Stroke, 2003;34:1699-1703.

23. Lees K, Zivin J, Ashwood T, et al., NXY-059 for acute ischemic stroke, N Engl J Med, 2006;354:588-600.

24. Shuaib A, Lees $K$, Lyden $P$, et al., NXY-059 for the treatment of acute ischemic stroke, N Engl I Med, 2007;357:562-71.

25. Bliss $T$, Guzman R, Daadi M, Steinberg G, Cell transplantation therapy for stroke, Stroke, 2007:38:817-26.

26. Moskowitz M, Lo E, Neurogenesis and apoptotic cell death Stroke, 2003;34:324-6.

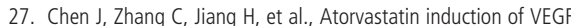
and BDNF promotes brain plasticity after stroke in mice, J Cereb Blood Flow Metab, 2005;25:281-90.

28. Wang L, Zhang Z, Zhang R, et al., Neurogenin 1 mediates erythropoietin enhanced differentiation of adult neural progenitor cells, J Cereb Blood Flow Metab, 2006;26:556-64.

29. Zhang $R$, Zhang Z, Zhang L, et al., Delayed treatment with silendafil enhances neurgenesis and improves functional recovery in aged rats after focal cerebral ischemia, J Neurosci Res, 2006;83:1213-19.

30. Kondziolka D, Steinberg G, Wechsler L, et al., Neurotransplantation for patients with subcortical motor stroke: phase 2 randomized trial, J Neurosurg, 2005;103:38-45

31. Kondziolka D, Wechsler L, Goldstein S, et al., Transplantation of cultured human neuronal cells for patients with stroke. Neurology, 2000;55:565-9.

32. Meairs $S$, Wahlgren $N$, Dirnagl U, et al., Stroke research priorities for the next decade $-\mathrm{A}$ representative view of the European scientific community. Summary of stroke research workshop, Brussels, 25 October 2005, Cerebrovasc Dis, 2005 22:75-82

\section{Stroke in Europe}

- Annually, 15 million people worldwide suffer a stroke. Of these, 5 million die and another 5 million are left permanently disabled, placing a burden on family and community.

- Stroke occurs in about $8 \%$ of children with sickle cell disease.

- Key modifiable risk factors include high blood pressure - for every 10 people who die of stroke, four could have been saved if their blood pressure had been regulated - and tobacco use. Atrial fibrillation, heart failure and heart attack are other important risk factors.

Predictors of Death from Stroke in Italy

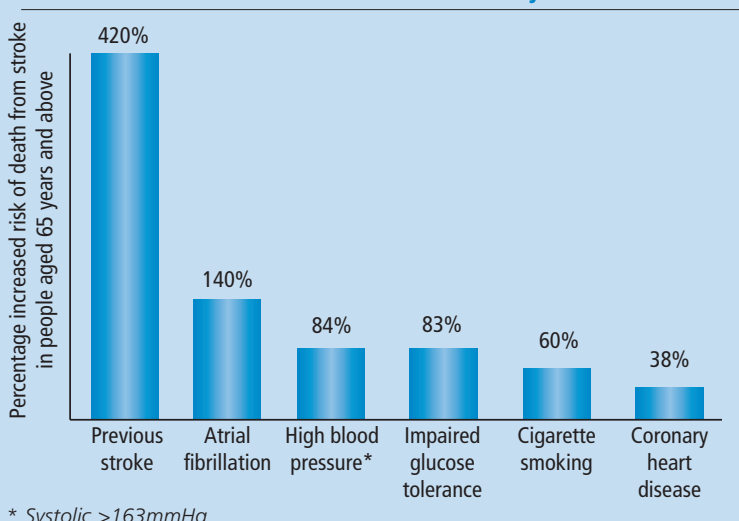

- The incidence of stroke is declining in many developed countries, largely as a result of better control of high blood pressure and reduced levels of smoking. However, the absolute number of strokes continues to increase because of the ageing population.

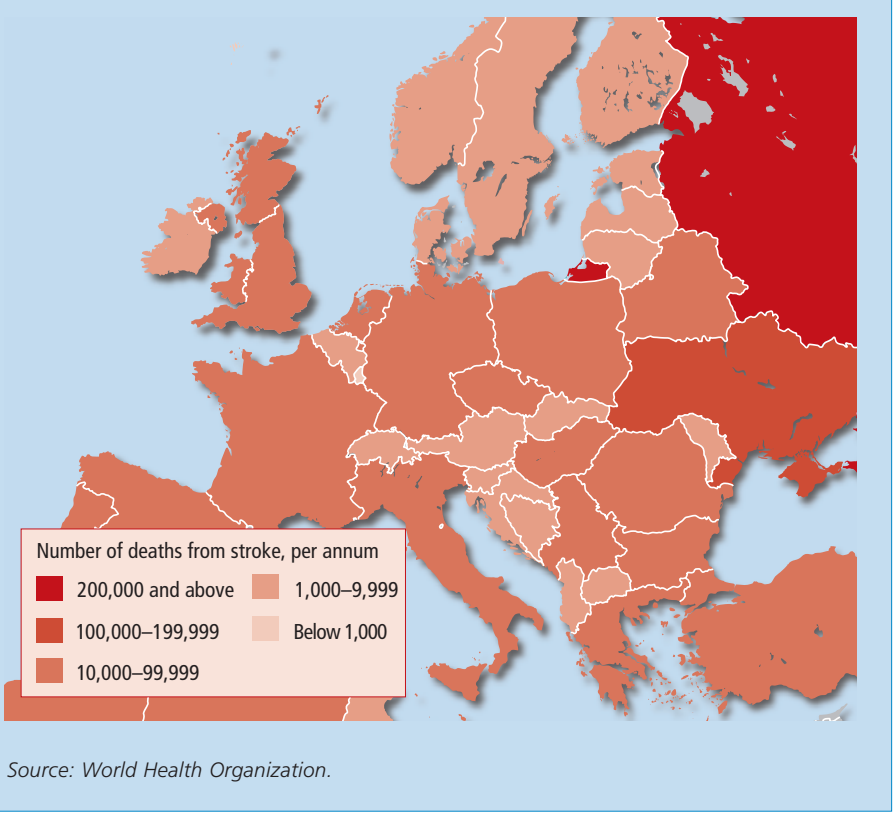

